\title{
On the growth of restricted integer partition functions
}

\author{
E. Rodney Canfield \\ Herbert S. Wilf \\ University of Georgia \\ University of Pennsylvania \\ Athens, GA 30602-7404 \\ Philadelphia, PA 19104-6395
}

November 7, 2018

\begin{abstract}
We study the rate of growth of $p(n, S, M)$, the number of partitions of $n$ whose parts all belong to $S$ and whose multiplicities all belong to $M$, where $S$ (resp. $M$ ) are given infinite sets of positive (resp. nonnegative) integers. We show that if $M$ is all nonnegative integers then $p(n, S, M)$ cannot be of only polynomial growth, and that no sharper statement can be made. We ask: if $p(n, S, M)>0$ for all large enough $n$, can $p(n, S, M)$ be of polynomial growth in $n$ ?
\end{abstract}

Subject classification number: 05A17 Primary

Keywords: integer partitions, asymptotic growth

\section{The question}

Let $S$ be a set of positive integers, and let $p_{S}(n)$ denote the number of partitions of the integer $n$ all of whose parts lie in $S$. For various sets $S$, the asymptotic growth rate of $p_{S}(n)$ is known, and the known rates lie in the range of polynomial growth to superpolynomialbut-subexponential rates.

For example, if $S$ consists of all positive integers then the celebrated theorem of Hardy, Ramanujan and Rademacher [3, 5] has given the complete asymptotic expansion, of which the first term is

$$
p_{S}(n) \sim \frac{1}{4 n \sqrt{3}} \exp \left(\pi \sqrt{\frac{2 n}{3}}\right) .
$$


As an example of a sparse set of parts, take $S=\left\{1,2,2^{2}, 2^{3}, \ldots\right\}$, the case of binary partitions. Then de Bruijn [2] found several terms of the asymptotics of the logarithm, which begins as

$$
\log p_{S}(2 n)=\frac{1}{2 \log 2}\left(\log \frac{n}{\log n}\right)^{2}(1+o(1)) .
$$

For a final example, suppose the set $S$ of allowable parts is finite. Then we have, say, $S=\left\{a_{1}<\ldots<a_{k}\right\}$, and we are dealing with "the money changing problem," a.k.a. "the problem of Frobenius." A result of Schur [6] holds that in this case $p_{S}(n)$ is of polynomial growth.

Theorem 1 (Schur) If $S=\left\{a_{1}<\ldots<a_{k}\right\}$, and $\operatorname{gcd}(S)=1$, then

$$
p_{S}(n) \sim \frac{n^{k-1}}{(k-1) ! a_{1} a_{2} \ldots a_{k}},
$$

and in particular, $p_{S}(n)>0$ for all large enough $n$.

We show here that if the set of allowable parts is infinite, no matter how sparse, then the partition function $p_{S}(n)$ must grow faster than every polynomial. We show also that this result is best possible in the sense that if $\epsilon(n)$ is any unbounded function of $n$ then there exists an infinite set $S$ of allowable parts such that $p_{S}(n)=O\left(n^{\epsilon(n)}\right)$.

We discuss also the situation in which we have an arbitrary set of allowable parts and an arbitrary set of allowable multiplicities.

\section{Preliminaries}

Lemma 1 Let $S=a_{1}<a_{2}<a_{3}<\ldots$ be a set of positive integers such that $\operatorname{gcd}(S)=1$. Then $S$ contains a finite coprime subset.

Proof. Let $g_{n}=\operatorname{gcd}\left(a_{1}, \ldots, a_{n}\right)$. Then $a_{1} \geq g_{1} \geq g_{2} \geq \ldots$, so $\exists i_{0}$ such that $\forall i>i_{0}$ : $g_{i}=1$. Indeed, if not then $\exists i_{0}$ such that $\forall i>i_{0}: g_{i}=g>1$. But then we would have $\operatorname{gcd}(S)=g>1$, a contradiction.

Lemma 2 The following two properties of a set $S$ of positive integers are equivalent:

1. for all sufficiently large integers $n$ we have $p_{S}(n)>0$

2. $\operatorname{gcd}(S)=1$. 
Proof. If $\operatorname{gcd}(S)=1$ then by Lemma $1 S$ contains a finite coprime subset $\bar{S}$. By Schur's theorem, $p_{\bar{S}}(n)>0$ for all large enough $n$, hence so is $p_{S}(n)$, and conclusion 1 holds. On the other hand, if $\operatorname{gcd}(S)>1$ then conclusion 1 is obviously false.

We remark that Lemma 2, whose proof we have given in order to keep this paper selfcontained, is a special case of a much more general result of Bateman and Erdös [1], who found the conditions on $S$ under which, for a fixed $k \geq 0$, almost all values of the $k$ th differences of $\left\{p_{S}(n)\right\}_{n=0}^{\infty}$ are strictly positive.

Next we will need a lemma that allows us to estimate the growth of $p_{S}(n)$ for arbitrary sets $S$ of parts. We will in fact prove a more general result, in which not only the set $S$ of allowable parts can be arbitrarily prescribed, but so can the set $M$ of allowable multiplicities of those parts.

Hence, let $S$ be a set of positive integers and let $M$ be a set of nonnegative integers such that $0 \in M$. Let $M(x), S(x)$ denote the respective counting functions of $M, S$. That is $M(x)=|\{\mu \in M: \mu \leq x\}|$, and likewise for $S(x)$. Finally we denote by $p(n ; S, M)$ the number of partitions of $n$ whose parts all belong to $S$ and the multiplicities of whose parts all belong to $M$.

Lemma 3 For the general partition function $p(n ; S, M)$ we have

$$
p(n ; S, M) \leq \prod_{a_{i} \in S} M\left(n / a_{i}\right)
$$

Further, there must exist at least one integer $r \leq n^{2}$ s.t.

$$
p(r ; S, M) \geq \frac{1}{n^{2}+1} \prod_{a_{i} \in S} M\left(n / a_{i}\right) .
$$

If also $p(n ; S, M)$ is a nondecreasing function of $n$ then we have the stronger statement that

$$
p(n ; S, M) \geq \frac{1}{n+1} \prod_{a_{i} \in S} M\left(\sqrt{n} / a_{i}\right) .
$$

Proof. Fix $n>0$ and consider the form

$$
\phi=m_{1} a_{1}+m_{2} a_{2}+m_{3} a_{3}+\ldots+m_{n} a_{n} .
$$

Now allow each of the $m_{i}$ to take any value that it wishes to take, subject to $m_{i} \in M$ and $m_{i} \leq n / a_{i}$. For each set of choices, the form $\phi$ is a partition of some integer $\leq n^{2}$, and all partitions of $n$ occur. 
The total number of values that the form takes, counting multiplicities, is

$$
\prod_{i} M\left(n / a_{i}\right)
$$

(Note that all terms with sufficiently large index $i$ are $=1$.) Since every partition of $n$ occurs, we find that

$$
p(n ; S, M) \leq \prod_{i} M\left(n / a_{i}\right) .
$$

Furthermore, since the average number of occurrences of the integers $\leq n^{2}$ is

$$
\frac{1}{n^{2}+1} \prod_{i} M\left(n / a_{i}\right),
$$

the second conclusion of the lemma is proved.

Let's test this with one or two examples. First take $M$ to be all nonnegative integers and $S$ to be all positive integers. then $M(x)=1+\lfloor x\rfloor$ and we find that $p(n ; S, M) \leq \prod_{i}(1+\lfloor n / i\rfloor)$. This is around $n^{n} / n$ !, which is roughly $e^{n}$, whereas the correct growth is around $e^{C \sqrt{n}}$. The lower bound is about $e^{n} / n^{2}$, so there exists an integer $r \leq n^{2}$ s.t. $p(r ; S, M) \geq \prod_{i}\lceil n / i\rceil / n^{2}$, which is about $e^{n} / n^{2}$. But indeed, if there is such an integer $r$, then since $p(n)$ is monotone, we can take $r=n^{2}$. Lemma 3 then says that $p\left(n^{2} ; S, M\right) \geq e^{n} / n^{2}$, or

$$
p(n ; S, M) \geq e^{\sqrt{n}} / n,
$$

which is reasonably sharp. For another example, in the case of binary partitions, the upper bound (6) yields the estimate

$$
\log p_{S}(2 n) \leq \log (2 n+1) \log _{2}(2 n) \sim \frac{(\log n)^{2}}{\log 2}
$$

which can be compared with (2).

\section{The growth of $p_{S}(n)$}

Theorem 2 Let $S$ be an infinite set of positive integers, and let $p_{S}(n)$ be the number of partitions of $n$ whose parts belong to $S$. Then $p_{S}(n)$ is of superpolynomial growth, that is, for every fixed $k$ the assertion $p_{S}(n)=O\left(n^{k}\right)$ is false. This result is best possible in the sense that if $\epsilon(n)$ is any function of $n$ that $\rightarrow \infty$, then we can find an infinite set $S$ such that $p_{S}(n)=O\left(n^{\epsilon(n)}\right)$. 
Proof. Let $S=\left\{1 \leq a_{1}<a_{2}<\ldots\right\}$. Then $g=\operatorname{gcd}(S) \leq a_{1}<\infty$, and the theorem is true for $S$ iff it is true for $S / g$. Hence we can, and do, assume w.l.o.g. that $\operatorname{gcd}(S)=1$.

Let $T \subseteq S$ be such a finite coprime subset, and put $k=|T|$. By Schur's theorem we have $p_{S}(n) \geq p_{T}(n) \sim C n^{k-1}$. But we can make $k$ arbitrarily large by adjoining elements of $S$ to $T$ since that adjunction preserves coprimality. Therefore $P_{S}(n)$ must grow superpolynomially.

For the second part of the theorem we use (44) with unconstrained multiplicities, i.e., with $M(x)=1+\lfloor x\rfloor$ for $x>0$. If we write $A(n)=\left|\left\{i: a_{i} \leq n\right\}\right|$ then (41) reads as

$$
\begin{aligned}
p_{S}(n) & \leq \prod_{i \geq 1}\left(1+\left\lfloor\frac{n}{a_{i}}\right\rfloor\right) \leq \prod_{a_{i} \leq n}\left(1+\frac{n}{a_{i}}\right) \leq n^{A(n)} \prod_{a_{i} \leq n}\left(\frac{1}{n}+\frac{1}{a_{i}}\right) \leq n^{A(n)} \prod_{a_{i} \leq n}\left(1+\frac{1}{a_{i}}\right) \\
& \leq n^{A(n)} \prod_{a_{i} \leq n} e^{1 / a_{i}} \leq n^{A(n)} e^{H_{n}}=O\left(n^{A(n)+1}\right),
\end{aligned}
$$

in which $H_{n}$ is the $n$th harmonic number. Evidently we can make this $O\left(n^{\epsilon(n)}\right)$ by taking the set $S$ to be sufficiently sparse.

\section{A partition function that grows slowly}

There are infinite sequences of allowable parts and multiplicities on which the partition function grows only polynomially fast, in fact it can even grow subpolynomially.

One such example is the case where the allowable parts are the sequence $\left\{2^{2^{j}}\right\}_{j=0}^{\infty}$ and the allowable multiplicities are

$$
\{0\} \cup\left\{2^{2^{j}}\right\}_{j=0}^{\infty} .
$$

In this case we have, in the notation above, $M(x)=1+\lfloor\lg \lg x\rfloor$, for $x \geq 4$, where "lg" is the $\log$ to the base 2 . Then by (6) we have

$$
\begin{aligned}
p(n ; S, M) & \leq \prod_{2^{2^{i}} \leq n / 4}\left(1+\left\lfloor\lg \lg \frac{n}{2^{2^{i}}}\right\rfloor\right) \leq \prod_{2^{2^{i}} \leq n / 4}\left(2\left\lfloor\lg \lg \frac{n}{2^{2^{i}}}\right\rfloor\right) \\
& \leq(\lg n)(\lg \lg n)^{\lg \lg n}
\end{aligned}
$$

which is of sub-polynomial growth. This argument fails if the parts and multiplicities are all of the powers of 2 .

The above argument can be generalized to give a fairly simple criterion, in terms of the sets of parts and multiplicities, for polynomial growth of the partition function. 


\section{$5 \quad$ Representing all large integers}

The example above shows that if the allowable multiplicities and parts are thin enough, even though they both are infinite sets, then the partition function can grow very slowly. But the example has the property some arbitrarily large integers are not represented at all. It may be that if we rule out such situations then the growth must be superpolynomial. We formulate this as

Unsolved problem 1: Let $S, M$ be infinite sets of nonnegative integers with $0 \notin S$, and let $p(n ; S, M)$ be the number of partitions of $n$ whose parts all lie in $S$ and the multiplicities of whose parts all lie in $M$. Suppose further that $p(n ; S, M)>0$ for all sufficiently large $n$. Must $p(n ; S, M)$ then be of superpolynomial growth?

Unsolved problem 2: Find necessary and sufficient conditions on $S, M$ in order that $p(n ; S, M)>0$ for all large enough $n$. Failing this, find as sharp as possible necessary conditions, and similarly sufficient conditions for this to happen.

Unsolved problem 3: Find necessary and sufficient conditions on $S, M$ in order that $p(n ; S, M)$ increase monotonically for all large enough $n$. Failing this, find as sharp as possible necessary conditions, and similarly sufficient conditions for this to happen).

\section{Monotonicity of the partition function}

With reference to unsolved problem 3 above, we consider the case where the set $S$ of allowable parts is finite and all multiplicities are allowed, i.e., the problem of Frobenius.

Theorem 3 Let $\{p(n)\}$ be generated by

$$
G(x) \stackrel{\text { def }}{=} \sum_{n \geq 0} p(n) x^{n}=\frac{1}{\prod_{i=1}^{k}\left(1-x^{a_{i}}\right)},
$$

where $\operatorname{gcd}\left(a_{1}, \ldots, a_{k}\right)=1$. The sequence $\{p(n)\}$ is strictly increasing for all sufficiently large $n$ if there does not exist a prime $p$ that divides all but one of the $a_{i}$ 's, i.e., iff every $(k-1)$-subset of the $a_{i}$ 's is coprime.

Proof: Evidently strict monotonicity holds from some point on iff

$$
(1-x) G(x)=\frac{1-x}{\prod_{i=1}^{k}\left(1-x^{a_{i}}\right)}
$$


has positive power series coefficients, from some point on. The partial fraction expansion of $(1-x) G(x)$ is of the form

$$
\begin{aligned}
(1-x) G(x)= & \frac{A_{0}}{(1-x)^{k-1}}+\frac{A_{1}}{(1-x)^{k-2}}+\ldots+\frac{B_{0}}{(1-\omega x)^{k_{1}}}+\frac{B_{1}}{(1-\omega x)^{k_{1}-1}}+\ldots \\
& +\frac{C_{0}}{(1-\zeta x)^{k_{2}}}+\frac{C_{1}}{(1-\zeta x)^{k_{2}-1}}+\ldots
\end{aligned}
$$

In the above, $\omega, \zeta$, etc. run through the primitive $p$ th roots of unity for each prime $p$ that divides one or more of the $a_{i}$ 's, and $k_{1}, k_{2}, \ldots$ are the number of $a_{i}$ 's that each of these primes divides. If no prime divides all but one of the $a_{i}$ 's then all of the $k_{i}$ 's are $\leq k-2$. If in that case we take the coefficient of $x^{n}$ on both sides of we have that

$$
p(n)-p(n-1)=A_{0}\left(\begin{array}{c}
n+k-2 \\
n
\end{array}\right)+O\left(n^{k-3}\right),
$$

which, since $A_{0}>0$, is positive for all large enough $n$, as claimed.

\section{$7 \quad$ A refinement of the lower bound}

Let's find a sharper lower bound for $p(n ; S)$, when $S$ is an infinite coprime set of admissible parts, and all multiplicities are availabe.

Let $A=\left\{1 \leq a_{1}<a_{2}<\ldots<a_{k}\right\}$ be a finite coprime subset of $S$. If we put $r^{\prime}(n ; A)=$ $\sum_{j \leq n} p(j ; A)$, then an inequality due to Padberg [4] states that

$$
r^{\prime}(n ; A) \geq \frac{(n+1)^{k}}{k ! a_{1} \ldots a_{k}}
$$

Now, for infinitely many $n$ we have $p(n ; A)=\max _{j \leq n} p(j ; A)$. Hence for such $n, r^{\prime}(n ; A) \leq$ $(n+1) p(n ; A)$, and therefore

$$
p(n ; A) \geq \frac{(n+1)^{k-1}}{k ! a_{1} \ldots a_{k}} .
$$

Next, extend the set $A$ by adjoining to it the next $h$ basis elements, to get a new coprime set

$$
A_{h}=\left\{a_{1}, a_{2}, \ldots, a_{k}, a_{k+1}, a_{k+2}, \ldots, a_{k+h}\right\} .
$$

If we apply (11) to $A_{h}$ we find that

$$
p(n ; S) \geq p\left(n ; A_{h}\right) \geq \frac{(n+1)^{k+h-1}}{(k+h) ! a_{1} a_{2} \ldots a_{k+h}} .
$$


Since $h$ is arbitrary we can optimize this inequality by defining $j=j(n)$ to be the least integer such that $j a_{j} \geq n$.

Theorem 4 Let $S$ be an infinite coprime set, and let $M$ consist of all nonnegative integers. Then for large enough $n$ we will have

$$
p(n ; S, M) \geq \frac{(n+1)^{j(n)-1}}{(j(n)) ! a_{1} a_{2} \ldots a_{j(n)}} .
$$

For example if $S$ consists of all positive integers we find for the classical partition function

that $p(n) \geq e^{2 \sqrt{n}} /\left(2 \pi n^{2}\right)$ for all large enough $n$, which can be compared to the bound (7), obtained earlier.

\section{References}

[1] Bateman, Paul T., and Erdős, Pál, Monotonicity of partition functions, Mathematika, London 3, (1956) 1-14.

[2] de Bruijn, N. G., On Mahler's partition problem, Nederl. Akad.Wetensch., Proc. 51, (1948) 659-669 = Indagationes Math. 10, 210-220 (1948).

[3] Hardy, G. H. and Ramanujan, S, Asymptotic formulæ in combinatory analysis, Proc. London Math. Soc. (2) 17 (1918), 75-115.

[4] Padberg, Manfred W., A Remark on "An Inequality for the Number of Lattice Points in a Simplex," SIAM J. Appl. Math. 20 (No. 4) 1971, 638-641.

[5] Rademacher, Hans, On the expansion of the partition function in a series, Ann. of Math. (2) 44, (1943). 416-422.

[6] I. J. Schur, Zur additiven zahlentheorie, Sitzungsberichte Preussische Akad. Wiss., Phys. Math. Kl. (1926), 488-495. 